\title{
Beam instability induced by rf deflectors in the combiner ring of the CLIC test facility and mitigation by damped deflecting structures
}

\author{
David Alesini, Caterina Biscari, Andrea Ghigo, and Fabio Marcellini \\ INFN Laboratori Nazionali di Frascati, P.O. Box 13, I-00044, Frascati (Roma), Italy \\ Roberto Corsini \\ CERN, Geneva, Switzerland \\ (Received 30 January 2010; published 7 February 2011)
}

\begin{abstract}
In the CTF3 (CLIC test facility 3) run of November 2007, a vertical beam instability has been found in the combiner ring during operation. After a careful analysis, the source of the instability has been identified in the vertical deflecting modes trapped in the rf deflectors and excited by the beam passage. A dedicated tracking code that includes the induced transverse wakefield and the multibunch multipassage effects has been written and the results of the beam dynamics analysis are presented in the paper. The mechanism of the instability was similar to the beam breakup in a linear accelerator or in an energy recovery linac. The results of the code allowed identifying the main key parameters driving such instability and allowed finding the main knobs to mitigate it. To completely suppress such beam instability, two new rf deflectors have been designed, constructed, and installed in the ring. In the new structures the frequency separation between the vertical and horizontal deflecting modes has been increased, changing the position of the rods inside the cells, and special antennas have been inserted to absorb the power released by the beam to the modes. The deflectors have been made in aluminum to reduce the costs and delivery time and have been successfully tested and installed in the ring. The design, the realization procedures, and the rf test results are illustrated.
\end{abstract}

\section{INTRODUCTION}

CTF3 [1] is the third test facility of the compact linear collider (CLIC) project [2,3]. The CTF3 main goals were, originally, to demonstrate the feasibility of the generation of a high beam current $(\sim 30 \mathrm{~A})$ electron pulse through the bunch recombination technique and its use to generate $30 \mathrm{GHz}$ rf power as well as the acceleration of a probe beam with $30 \mathrm{GHz}$ rf cavities at very high gradient $(\sim 150 \mathrm{MV} / \mathrm{m})$. During the commissioning period, the target frequency generation has been changed from 30 to $12 \mathrm{GHz}$ and from 150 to $100 \mathrm{MV} / \mathrm{m}$ [4].

The frequency multiplication by the novel bunch interleaving technique is one of the most important issues to be tested. In CTF3 a long $(1.12 \mu \mathrm{s})$ train of $20 \mathrm{~cm}$ spaced short bunches (drive beam) is converted into a short (140 ns) train of $2.5 \mathrm{~cm}$ spaced bunches [1]. The conceptual layout of CTF3 is shown in Fig. 1.

In the first stage the pulse is compressed by a factor of 2 by means of a transverse rf deflector (RFD) at $1.499 \mathrm{GHz}$ and a $42 \mathrm{~m}$ circumference delay loop (DL). An $84 \mathrm{~m}$ circumference combiner ring (CR) is used in the second stage to further compress the pulse by a factor 4 .

The process of beam compression in the DL is illustrated in Fig. 2(a). The beam coming from the LINAC is composed by 1680 bunches in an alternate sequence of eight "even" and "odd" subtrains, which have a phase difference of $180^{\circ}$ with respect to the $1.499 \mathrm{GHz}$ of the RFD.
The length of each subtrain is $140 \mathrm{~ns}$. The rf deflector kicks, with the same amplitude but opposite sign, the incoming even and odd trains. The even trains are injected into the DL and, by properly setting the DL circumference, after one turn, they are extracted and interleaved with the following odd trains. After the DL the long $1.12 \mu$ s train is a sequence of four $140 \mathrm{~ns}$ subtrains spaced by gaps of $140 \mathrm{~ns}$ and each train is composed by 420 bunches.

The second stage of the bunch train compression in CTF3 is realized in the CR. The process of bunch train compression in the CR is illustrated in Fig. 2(b) and widely discussed in $[1,5]$. The bunch train recombination in the $\mathrm{CR}$ is performed using two traveling wave (TW) RFDs working at $f_{\text {rf }}=2.99855 \mathrm{GHz}$ already built [6] and successfully tested in the CTF3 preliminary phase at low current [5]. The structures deflect in the horizontal plane with the same maximum field amplitude. The horizontal betatron phase advance between them is $\pi$ and this allows creating a time-dependent local closed bump of the reference orbit, as described in the following.

The bunches of the first $140 \mathrm{~ns}$ train (coming from the DL) receive the maximum on-crest kick from the first rf deflector and are injected into the closed orbit of the CR. Since the length of the ring is an integer number rf wavelength plus (or minus) a quarter of wavelength, and since the distance between the two rf deflectors is an integer number of wavelengths, after one turn, the bunches pass the second and first deflectors at the zero crossing of the rf 


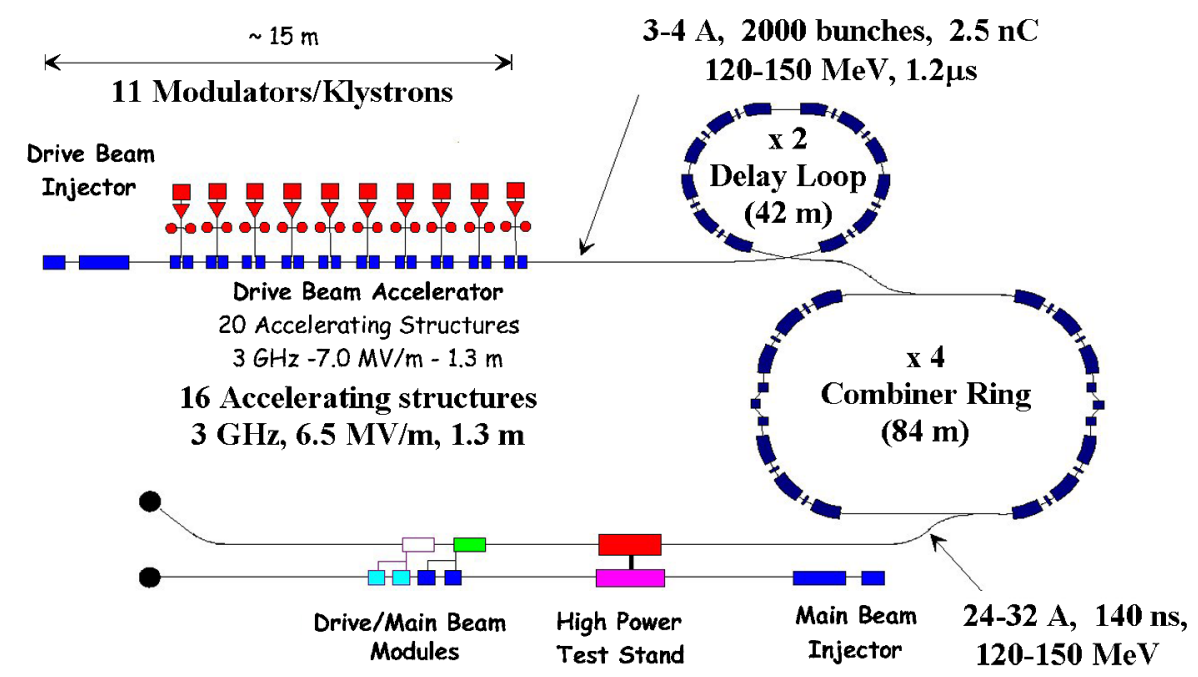

FIG. 1. Conceptual layout of CTF3.

deflecting field remaining on the unperturbed closed orbit. The second train is then injected into the ring and recombined with the first one. In the second turn, the first train of bunches is kicked in the opposite direction by the second deflector and their orbits are closed by the kick of the first deflector since the horizontal betatron phase advance between the deflectors is $\pi$. Similarly, the second train of bunches arrives at the zero crossing, and the third train is injected. After the third turn, the first train of bunches arrives again at the zero crossing, the second train of bunches is kicked away from the septum, the third train of bunches is also at the zero crossing, and the fourth train is injected. The four trains are now combined into one single train and the initial bunch spacing is reduced by a factor 4 .

The main beam parameters at the injection/extraction of the CR are reported in Table I.

In the November 2007 run at high current a vertical beam instability in the CR was observed during operation [7]. As an example the scope traces from a ring beam position monitor (BPM) are reported in Fig. 3 in the case of a single train of 420 bunches circulating in the CR. The plot shows, from top to bottom, the circulating beam current and the vertical and horizontal signals. A fast growing oscillation in the vertical plane is clearly visible and well correlated with beam losses. The oscillation amplitudes are of the order of $10 \mathrm{~mm}$. Such behavior is not present in the horizontal plane. Because of this instability the recombination process with all 1680 bunches was not possible.

The phenomenology of such instability is described in detail in [7] and can be summarized as follows: (i) the profile of the vertical oscillation as a function of the bunch positions did not change shot by shot, in other words the amplitude and phase of the oscillations were remarkably stable, every pulse, showing almost exactly the same trace on the scope; (ii) the measured $\Delta$ frequency of the oscil- lation with respect to $f_{\mathrm{rf}}$ was $\sim 48 \mathrm{MHz}$; (iii) the instability was stronger increasing the train length or the bunch charges; (iv) changing the temperature of the deflectors by $+8^{\circ} \mathrm{C}$ did not change the scenario; (v) the instability occurred both in the case of a single and of recombined train; and (vi) a better steering inside the deflectors seemed to yield a weaker instability, even if no systematic study was done.

All these characteristics, in particular the fact that the measured $\Delta$ frequency of the oscillation was about $48 \mathrm{MHz}$ shifted with respect to $f_{\text {rf }}$, were the first strong hint that the instability was due to the vertical deflecting modes (VDMs) trapped in the RFDs and excited by the beam. As described in detail in the first section of the paper, such modes have been in fact decoupled in frequency from the main horizontal mode by inserting metallic rods in the deflector cells, in order to avoid mode conversion of the injected power.

A detailed study of the wakefields induced by these modes and their effect on beam dynamics is presented in the first section of the paper. The analysis showed that the instability could be completely canceled with a strong reduction of the quality factors of the trapped modes and with a strong frequency shift of such modes with respect to the horizontal deflecting polarity (working mode). For these reasons, new RFDs have been designed and constructed. In the second section we illustrate the design of the new RFDs. The realization procedure and the rf tests are discussed in the third section. A brief description of the machine operation with the new devices is reported in the fourth section.

\section{VERTICAL MODES IN RF DEFLECTORS AND TRACKING CODE RESULTS}

The previous RFDs installed in the CR were TW devices [6] deflecting the beam in the horizontal plane (Fig. 4). 
(a)

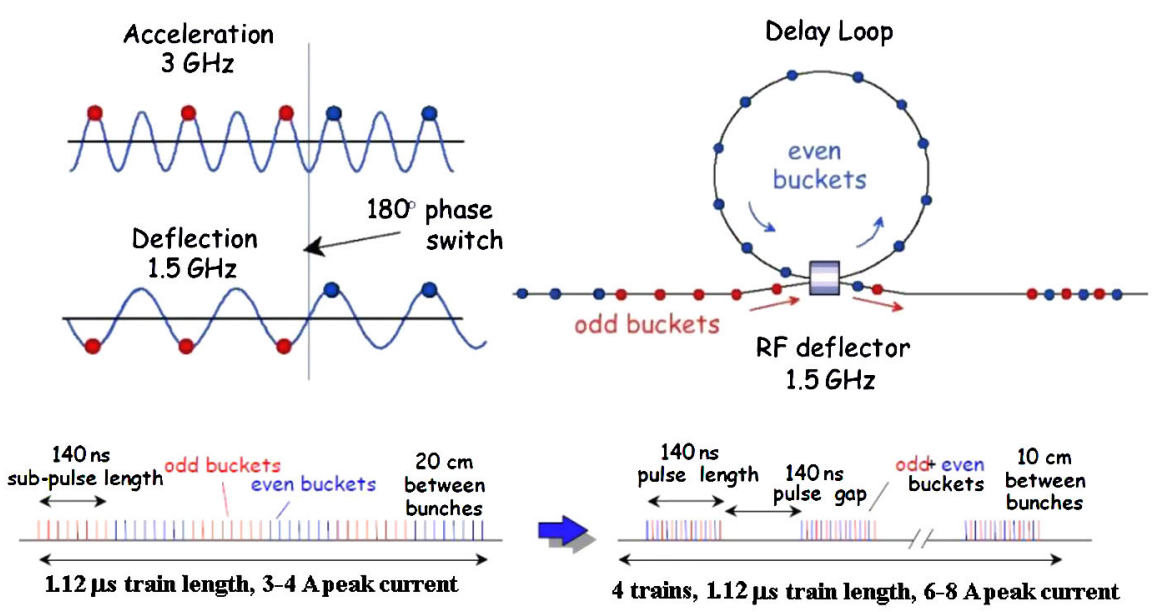

(b)
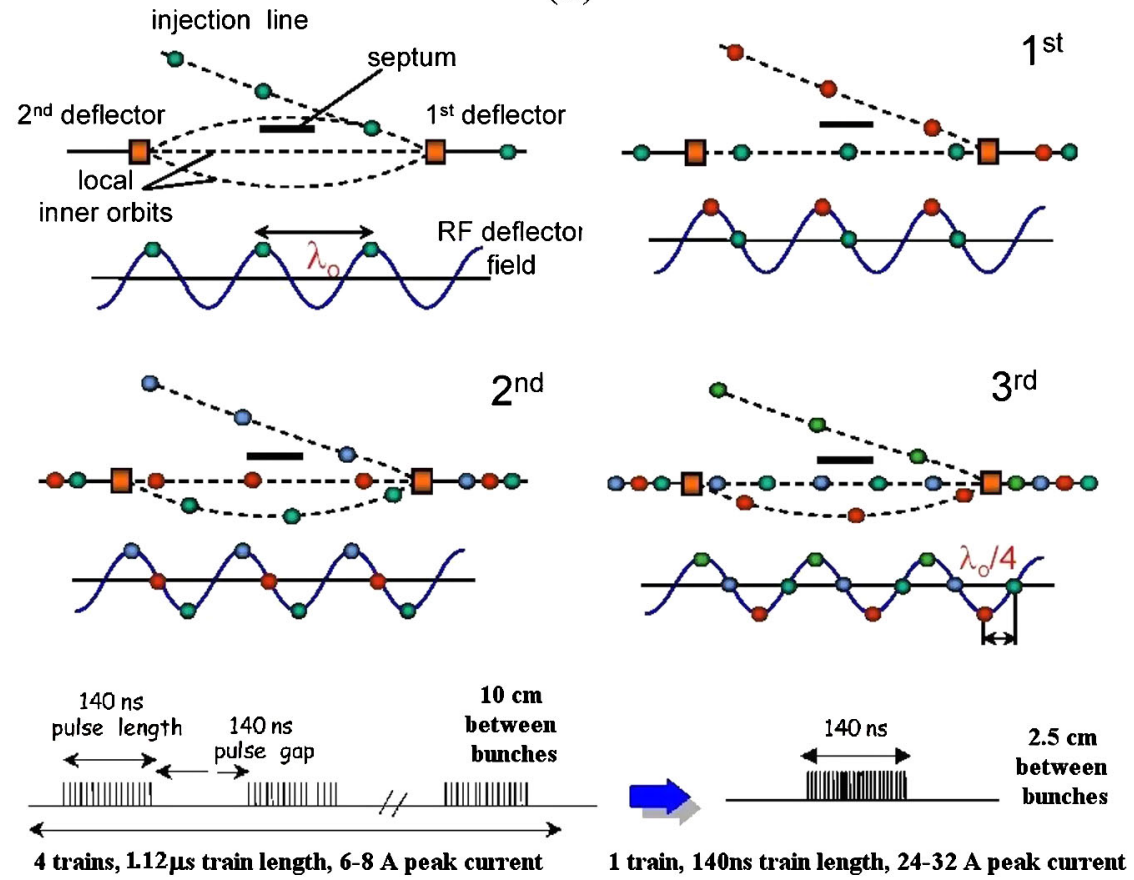

FIG. 2. (a) Sketch of the bunch frequency multiplication in the DL (a) and in the CR (b).

The main parameters of the structures are reported in Table II. Two metallic rods have been inserted into each cell to separate in frequency the deflecting mode with vertical polarity towards higher frequencies. The dimensions and position of the rods have been chosen to fix the polarity of the horizontal mode, avoiding tilt of the working polarity through the deflector and avoiding the excitation of the vertical mode by the beam power spectrum line at $2.99855 \mathrm{GHz}$. These vertical modes are not coupled to the input and output couplers. To evaluate the effect on beam dynamics due to the transverse wakefields induced by VDMs, the resonant frequencies $\left(f_{\text {res }}\right)$, quality factors
$(Q)$, and transverse shunt impedances $\left(R_{T}\right)^{1}$ of each VDM have been calculated by HFSS [8]. The results are plotted in Fig. 5. It is easy to note that there is a "dominant" mode (in terms of $R_{T}$ ) corresponding to the mode with a $\sim 2 \pi / 3$

${ }^{1}$ We define the transverse shunt impedance by the formula

$$
R_{T}=\frac{\left|\int_{\text {cavity }}\left(c B_{x}+E_{y}\right) e^{i \omega_{\mathrm{r} z} / c} d z\right|^{2}}{2 P_{\text {diss }}},
$$

where $B_{x}$ and $E_{y}$ are the magnetic and electric transverse field components, $z$ is the beam traveling direction, and $P_{\text {diss }}$ is the total dissipated power in the cavity. 
TABLE I. Main CTF3 parameters at the injection/extraction of the CR.

\begin{tabular}{|c|c|c|}
\hline \multicolumn{2}{|l|}{ Energy $(E)$} & $120-150[\mathrm{MeV}]$ \\
\hline \multicolumn{2}{|c|}{ Circumference length $(L)$} & $84 \mathrm{~m}$ \\
\hline \multicolumn{2}{|c|}{ Bunch charge $\left(Q_{b}\right)$} & $2.33[\mathrm{nC}]$ \\
\hline \multicolumn{2}{|c|}{ Bunch length $\left(\sigma_{z}\right)$} & $1.5-2.5[\mathrm{~mm}]$ \\
\hline \multicolumn{2}{|c|}{ Beam emittance $^{\mathrm{a}}(\varepsilon)$} & $0.4[\mathrm{~mm} \mathrm{mrad}]$ \\
\hline \multicolumn{2}{|c|}{ Number of bunches per pulse $\left(N_{b}\right)$} & 1680 \\
\hline \multirow[t]{3}{*}{ Injection } & Pulse duration $\left(\tau_{\mathrm{CR} \text { inj }}\right)$ & $1.12[\mu \mathrm{s}]$ \\
\hline & Number of trains per pulse $\left(N_{\text {pinj }}\right)$ & 4 \\
\hline & Bunch separation $\left(\tau_{B}\right)$ & $0.33[\mathrm{~ns}]$ \\
\hline \multirow[t]{3}{*}{ Extraction } & Pulse duration $\left(\tau_{\mathrm{CR} \text { extr }}\right)$ & $140[\mathrm{~ns}]$ \\
\hline & Number of trains per pulse $\left(N_{\text {pextr }}\right)$ & 1 \\
\hline & Bunch separation $\left(\tau_{A}\right)$ & $0.083[\mathrm{~ns}]$ \\
\hline
\end{tabular}

${ }^{\mathrm{a}}$ The definition of beam emittance used in this evaluation is $\varepsilon=$ $\sqrt{\left\langle x^{2}\right\rangle\left\langle x^{\prime 2}\right\rangle-\left\langle x x^{\prime}\right\rangle^{2}}$, where $x, x^{\prime}$ are the position and angle of particles in the transverse phase space and \langle\rangle is the average value over the bunch. The reported value is referred to $120 \mathrm{MeV}$.

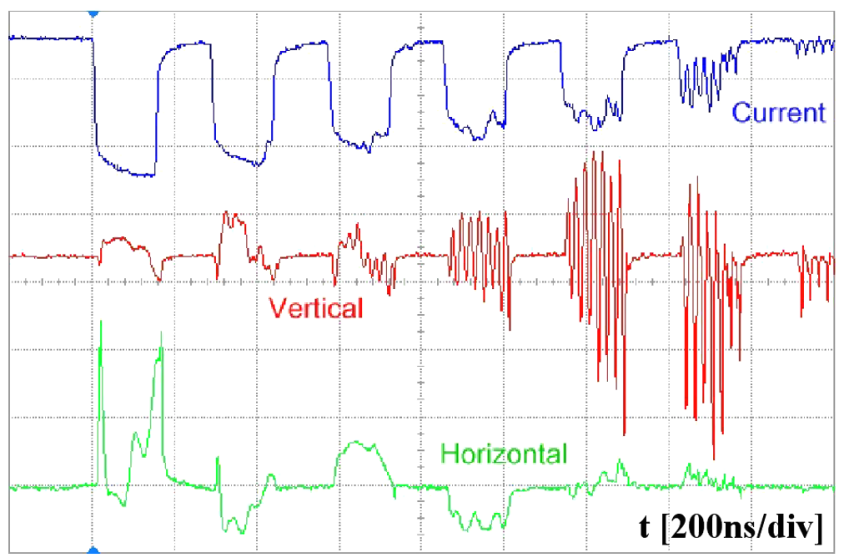

FIG. 3. Scope traces from a ring BPM showing the CR vertical instability. The plot shows, from top to bottom, the circulating beam current in the case of 400 bunches and the vertical and horizontal signals. The vertical scale for the current signal is $\approx$ $2 \mathrm{~A} /$ division, the vertical scale for the vertical signal is $\approx$ $4 \mathrm{~mm} /$ division, and for the horizontal one is $\approx 2 \mathrm{~mm} /$ division.

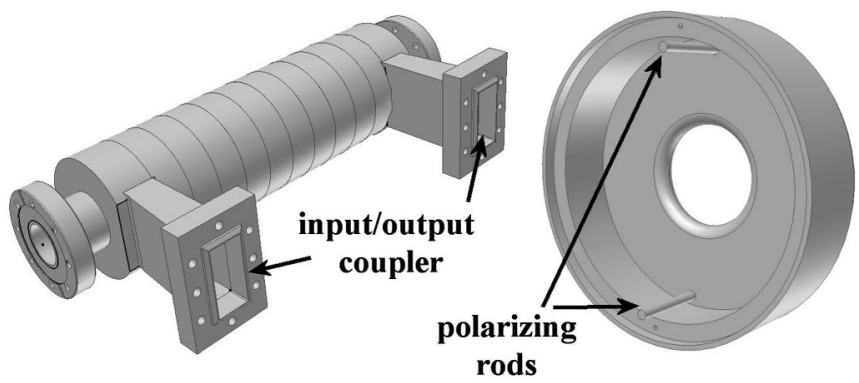

FIG. 4. Mechanical drawing of the old RFD of the CTF3 CR and detail of the single deflecting cell with polarizing rods.
TABLE II. Combiner ring rf deflector parameters.

\begin{tabular}{ll}
\hline \hline Frequency $(f)$ & $2.99855[\mathrm{GHz}]$ \\
\hline Cell length $(D)$ & $33.33[\mathrm{~mm}]$ \\
Cell radius $(b)$ & $56.01[\mathrm{~mm}]$ \\
Iris internal radius $(a)$ & $21.43[\mathrm{~mm}]$ \\
Iris thickness $(t)$ & $9.53[\mathrm{~mm}]$ \\
Number of active cells $\left(N_{c}\right)$ & 10 \\
Phase advance per cell $(\beta D)$ & $2 \pi / 3$ \\
Total deflector length $(L)$ & $33[\mathrm{~cm}]$ \\
Group velocity $\left(v_{g} / c\right)$ & -0.0237 \\
Filling time $\left(\tau_{F}\right)$ & $47[\mathrm{~ns}]$ \\
Shunt impedance per unit length $\left(r_{s}\right)$ & $18.1[\mathrm{M} \Omega / \mathrm{m}]$ \\
$r_{s} / Q$ & $1425 \Omega / \mathrm{m}$ \\
Nominal (maximum) input power & $\sim 2(\sim 10)[\mathrm{MW}]$ \\
Nominal $($ maximum) transverse voltage & $\sim 0.9(\sim 2)[\mathrm{MV}]$ \\
$E_{\text {surf }}$ at $P_{\text {in }}=10 \mathrm{MW}$ & $\sim 21[\mathrm{MV} / \mathrm{m}]$ \\
$H_{\text {surf }}$ at $P_{\text {in }}=10 \mathrm{MW}$ & $\sim 65[\mathrm{kA} / \mathrm{m}]$ \\
Maximum repetition rate & $10[\mathrm{~Hz}]$ \\
rf operating pulse length & $\sim 1.5[\mu \mathrm{s}]$ \\
\hline \hline
\end{tabular}

phase advance per cell. In the following calculations we have considered the contribution of this dominant mode only (whose parameters are reported in Table III). It is straightforward to extend all the obtained results to the more general case of multimodes.

The general expression of the transverse voltage (as a function of time) induced by a pointlike charge $(q)$ passing into the deflector with a vertical offset ( $y$ ) and exciting a resonant vertical mode is given by [9-11]

$$
V_{T}(\tau) \cong q \frac{\omega_{\text {res }}^{2}}{c} \frac{R_{T}}{Q} y e^{-\left(\omega_{\text {res }} / 2 Q\right) \tau} \sin \left(\omega_{\text {res }} \tau\right),
$$

where $\omega_{\text {res }}$ and $Q$ are the angular rf frequency and the quality factor of the resonant mode, respectively. The induced wakefield has a sine dependence and it is
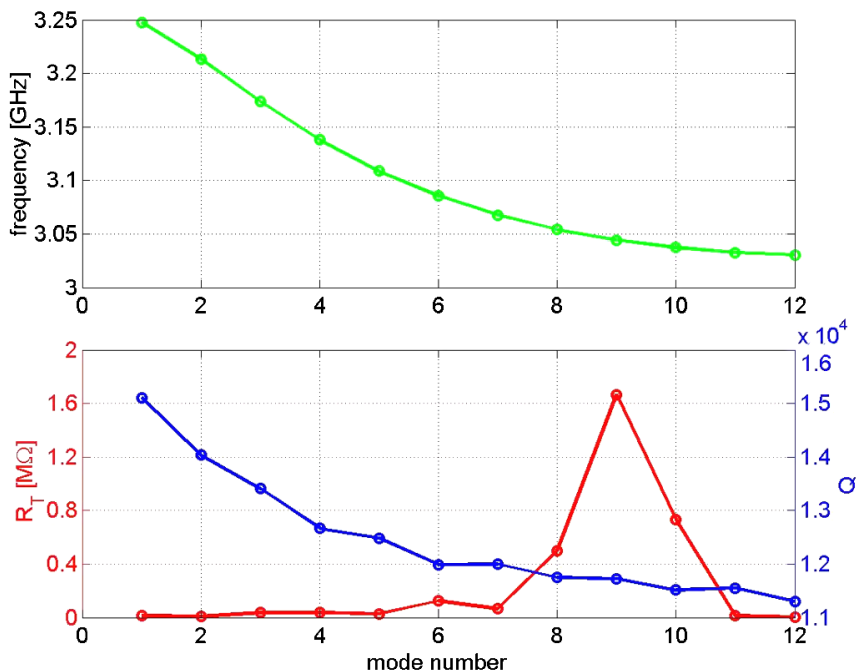

FIG. 5. Resonant frequency, quality factor, and transverse shunt impedance of the vertical trapped modes in the RFDs. 
TABLE III. Parameters of the dominant trapped vertical mode.

\begin{tabular}{ll}
\hline \hline$Q$ & 11500 \\
\hline$f_{\text {res }}$ & $3.0443 \mathrm{GHz}$ \\
$R_{T}$ & $1.6 \mathrm{M} \Omega$ \\
\hline \hline
\end{tabular}

the so-called 90 deg out-of-phase wake. It means that, if the mode is excited perfectly on resonance by the beam train, ${ }^{2}$ the bunches of the same train do not receive a net deflecting kick since they cross the cavity at the zero crossing of the transverse voltage. This is extensively described in [9].

If we consider the simple case of a single train injected in the $\mathrm{CR}$, it is straightforward to note that, since the separation between bunches is $1 / f_{\mathrm{rf}}$, the vertical deflecting mode is not excited perfectly on resonance by the beam train since its resonant frequency differs from $f_{\text {rf }}$ by 45.8 MHz. This may result in a net deflecting kick on bunches of the same train still in the first passage through the deflector. In the other passages into the deflector, one has to consider that, because of the CR length, the bunches arrive into the deflector with different phases with respect to the excited deflecting voltage and the situation becomes more complicated.

A dedicated tracking code [12] has been written to study the multibunch multipassage effects. The scheme of the tracking code is given in Fig. 6. In the code each bunch, represented as a macroparticle, enters the first deflector with a given vertical orbit $\left(Y_{\text {in } 1}\right)$, interacts with the wakefield left by the bunches ahead, contributes to the wake, and exits from the deflector. The bunch is then transported to the other deflector by the $\mathrm{CR}$ transport matrix $\left(M_{\mathrm{CR}}\right)$, enters the second RFD with a given vertical orbit $\left(Y_{\text {in2 }}\right)$ plus the perturbation given by the residual oscillation induced by the wakes in the first RFD, interacts with the rf field and wakes of this second device, and so on. At the end each macroparticle ends up with vertical $\Delta$ positions with respect to the original orbit $\left(y_{\text {out }}, y_{\text {out }}^{\prime}\right)$ given by the corresponding values of the Courant-Snyder invariant $\left(I_{\text {out }}\right)$. The tracking allows studying the distribution of $I_{\text {out }}$ for all bunches and its dependence on the resonant mode properties and ring optical functions. Since all the results scale with the charge per bunch $(q)$ and beam energy $\left(E_{0}\right)$, we fixed $q=2.33 \mathrm{nC}$ (nominal CTF3 charge) and $E_{0}=100 \mathrm{MeV}$ (beam energy during the November 2007 run). As an example the output vertical positions, angles, and $I_{\text {out }}$ normalized to the nominal CR emittance ( $\varepsilon=0.4 \mathrm{~mm} \mathrm{mrad})$ of one train of bunches after four turns are reported in Fig. 7 assuming the parameters reported in the figure caption $(\beta, \alpha$, and $\phi$ are the usual optical functions at the RFDs). As expected (because the beam excites the vertical mode polarity), the fast-Fourier transform (FFT) of the vertical oscillations have frequency

\footnotetext{
${ }^{2}$ This is, for example, the case in which the separation between bunches is $1 / f_{\text {res. }}$.
}

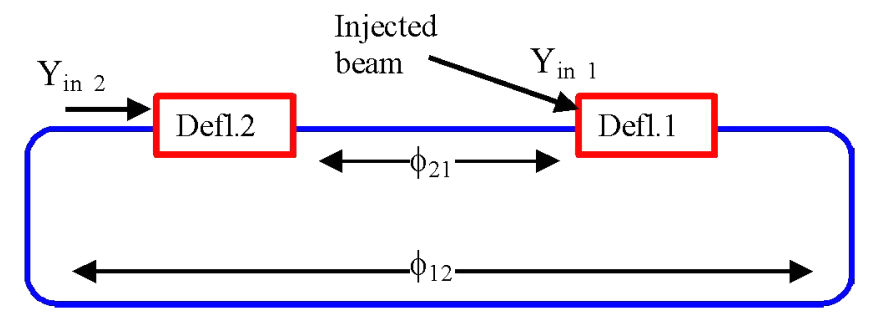

FIG. 6. Tracking code scheme.

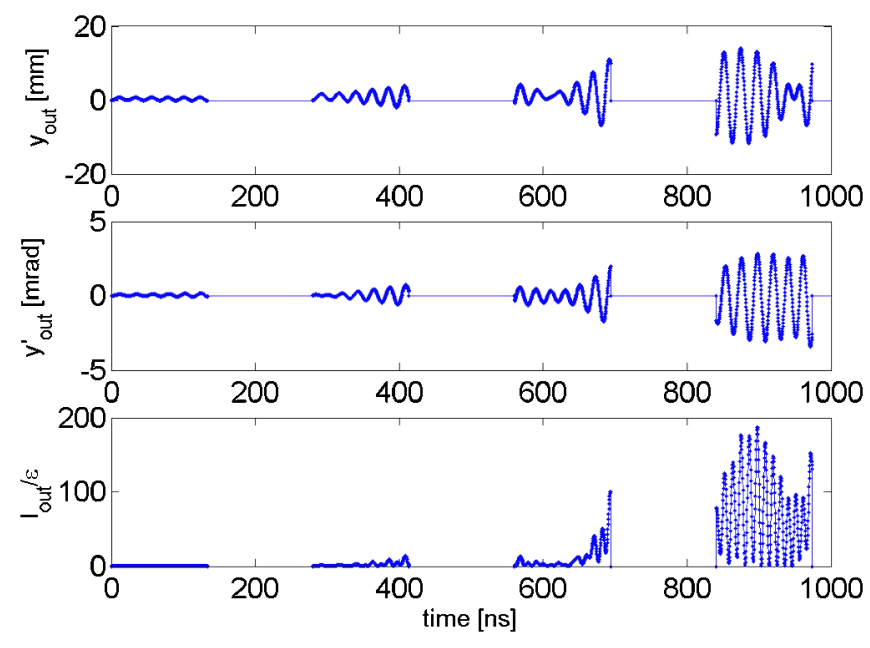

FIG. 7. Output vertical positions, angles, and $I_{\text {out }} / \varepsilon$ of a circulating train after four turns $\left(Y_{\text {in } 1}=2 \mathrm{~mm}, Y_{\text {in2 }}=0, \beta_{y 1}=\right.$ $\beta_{y 2}=5 \mathrm{~m}, \alpha_{y 1}=\alpha_{y 2}=0, \varphi_{12}=\varphi_{21}=30 \mathrm{deg}, N_{\text {bunches }}=$ $\left.400, N_{\text {turn }}=4\right)$.

components centered around $46 \mathrm{MHz}^{3}$ as shown in Fig. $8(\mathrm{~b}){ }^{4}$

The instability induced by the vertical mode can be also analyzed in the frequency domain. In this case the resonance is excited by the beam spectrum lines shown in Fig. 8(a) and referred to the case of a single train of 400 bunches that performs an infinite number of turns in the $\mathrm{CR}$. In the frequency domain the instability induced by this vertical mode can be "seen" by a classical interaction between a single resonant mode and a vertical beam coupling impedance. The beam spectrum lines excite this mode that, interacting with beam, induces an oscillation whose spectrum components are centered on the resonant frequency of the vertical mode itself.

The amplitudes of the induced oscillations are comparable with those measured through the BPM (and, as example, reported in Fig. 3). Nevertheless the signals cannot be directly compared with the simulation results since the amplitudes of the induced oscillations depend on the "real" orbit in the deflectors and also by the nonlinear response of the BPMs in the case of large oscillation

\footnotetext{
${ }^{3}$ This is equal to the difference between $f_{\text {rf }}$ and $f_{\text {res }}$.

${ }^{4} f_{\text {rev }}$ is the revolution frequency equal to $\sim 3.57 \mathrm{MHz}$.
} 

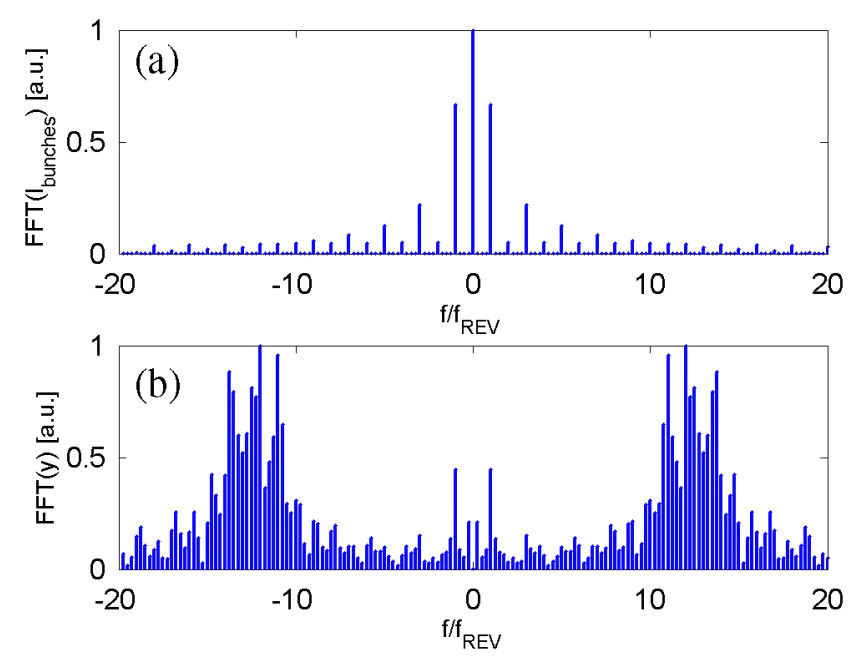

FIG. 8. (a) Beam spectrum lines of a single train of 400 bunches that perform an infinite number of turns in the CR. (b) FFT of the vertical oscillation signal of Fig. 7.

amplitudes. Moreover, in the simulations we have considered the dominant mode only while also the nearest modes (see Fig. 5) can contribute to the final oscillation profiles.

The average $I_{\text {out }} / \varepsilon$ in the last turn (calculated over the bunch train) as a function of the CR vertical phase advance $\left(\phi_{12}\right)$ and vertical $\beta$ function at the RFDs is given in Fig. 9 for different vertical phase advances between the two RFDs $\left(\phi_{21}\right)$.

Figure 10 shows the average $I_{\text {out }} / \varepsilon$ in the last turn as a function of the mode resonant frequency and quality factor. In the first case the average $I_{\text {out }} / \varepsilon$ has a periodic behavior due to the interaction between the finite bandwidth resonance and the periodic spectrum of the circulating beam and the periodicity is exactly equal to the revolution
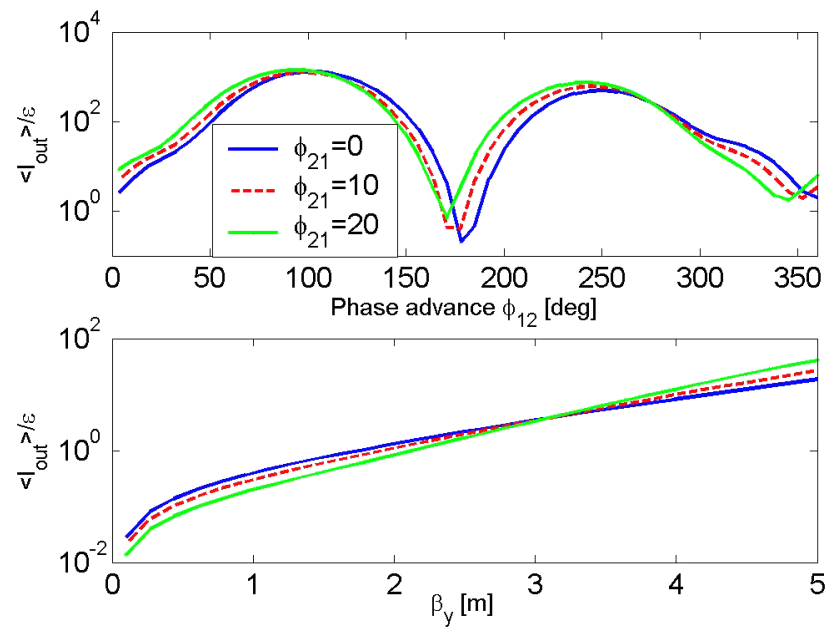

FIG. 9. Average $I_{\text {out }} / \varepsilon$ in the last turn (calculated over the bunch train) as a function of the CR vertical phase advance $\left(\varphi_{12}\right)$ and vertical $\beta$ function at the RFDs $\left(Y_{\text {in } 1}=2 \mathrm{~mm}, Y_{\mathrm{in} 2}=0\right.$, $\beta_{y 1}=\beta_{y 2}=5 \mathrm{~m}, \quad \alpha_{y 1}=\alpha_{y 2}=0, \quad \varphi_{12}=\varphi_{21}=30 \mathrm{deg}$, $N_{\text {bunches }}=400, N_{\text {turn }}=4$ ).

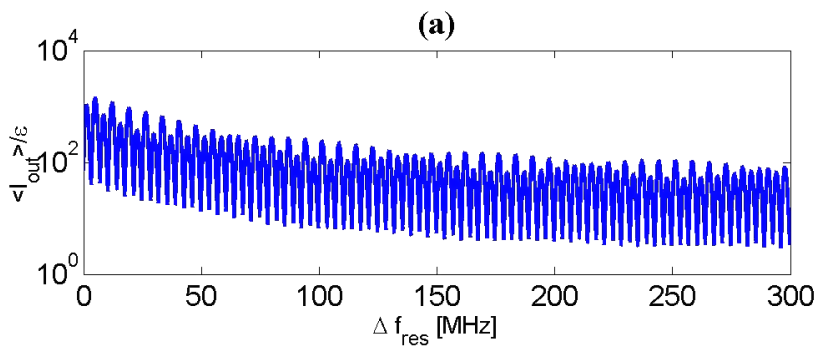

(b)

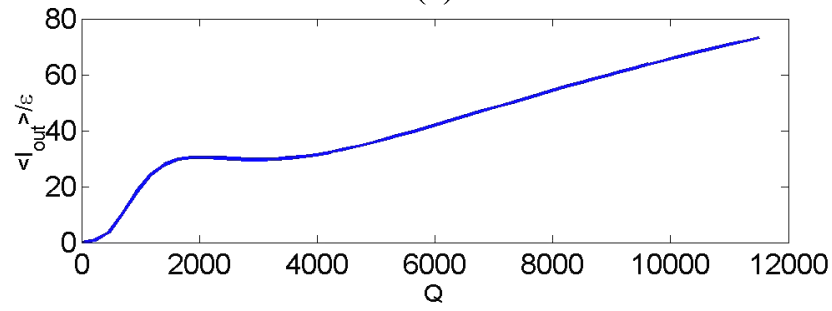

FIG. 10. Average $I_{\text {out }} / \varepsilon$ in the last turn (calculated over the bunch train) as a function of the mode resonant frequency and quality factor $\left(Y_{\mathrm{in} 1}=2 \mathrm{~mm}, Y_{\mathrm{in} 2}=0, \beta_{y 1}=\beta_{y 2}=5 \mathrm{~m}, \alpha_{y 1}=\right.$ $\left.\alpha_{y 2}=0, \varphi_{12}=\varphi_{21}=30 \mathrm{deg}, N_{\text {bunches }}=400, N_{\text {turn }}=4\right)$.

frequency $f_{\text {rev }}$. Similar results can be obtained considering different train lengths or considering the recombination.

It is straightforward to note that in the case with $\Delta f_{\text {res }}=$ 0 we are in full coupling with the vertical deflecting mode. In this case in the first turn the bunches of the same train do not experience a net deflecting kick because of the $90 \mathrm{deg}$ out-of-phase wake, but, in the second turn, they arrive into the deflector with a different phase because of the ring length and they are in full coupling with the excited deflecting mode.

Concerning the behavior of the amplitude of the instability as a function of the $Q$ factor, it depends on the machine parameters (optical parameters, optical $\beta$ functions, number of bunches) and, in different cases, we can have different behaviors. In all cases we verified that a reduction of the $Q$ factor below 100 suppresses the instability.

From the previous plots it was possible to conclude that this strong instability was caused by a few $\mathrm{mm}$ off-axis beam passage into the deflectors (1-2 $\mathrm{mm}$ was the order of magnitude of the orbit inside these devices in November 2007) and that a better orbit control inside these structures can reduce the instability. A vertical tune near half integer reduces the instability effects also ${ }^{5}$ as well as the reduction of the vertical $\beta$ functions at the RFDs. Changing the mode resonant frequencies by few hundred $\mathrm{kHz}$ (for example, by changing the temperature of the RFD

\footnotetext{
${ }^{5}$ This can be intuitively understood because, in the case of a half integer tune, the vertical kicks given by the wakefields do not change the vertical beam offset at the RFD after one turn. On the contrary, the maximum instability occurs when the vertical kick is completely transformed into vertical displacements (tune near 0.25 or 0.75 ).
} 
by few degree) did not help much because a detuning of the vertical mode of few hundred of $\mathrm{MHz}$ is needed to have relevant effects. In this case, in fact, the vertical resonance is no more excited by the beam power spectrum lines. A strong reduction of the $Q$ factors of the modes was the most effective solution to reduce the instability.

This type of instability has been already carefully investigated in linac [10] and energy recovery linacs [11] also with an analytical approach. Similar results to those previously illustrated here can be found in the cited references.

\section{NEW RF DEFLECTOR DESIGN}

The beam dynamics simulations clearly show that, in order to have a reduction of the oscillation amplitude of a factor 10 , a $\Delta f_{\text {res }} \approx 300 \mathrm{MHz}$ is needed. A further frequency shift gives quite small improvements. On the other hand, a reduction of the mode quality factors below 100 completely suppresses the instability because, in this case, the average invariant is comparable with the beam emittance itself. Therefore the combination of a frequency shift of $300 \mathrm{MHz}$ with a $Q$ factor below 100 guarantees that the instability is completely canceled in all operative conditions. These results guided the design of the new deflectors [13].

The frequency shift has been increased by moving the rods in each cell towards the center of the structure as shown in Fig. 11(b) while the second goal has been achieved modifying one of the two rods of each cell (except those of the input and output couplers) into a damping antenna loop connected to an external load [Fig. 11(c)]. The whole design has been done using HFSS [8]. The position and diameter of the rods have been chosen in order to have a strong frequency shift of the vertical polarity $(\approx 300 \mathrm{MHz})$. A further increase of the diameter and a further displacement of the rods toward the center of the structure did not give a significant shift and, on the other hand, could result in a perturbation of the working deflecting mode. The final distance of each rod with respect to the

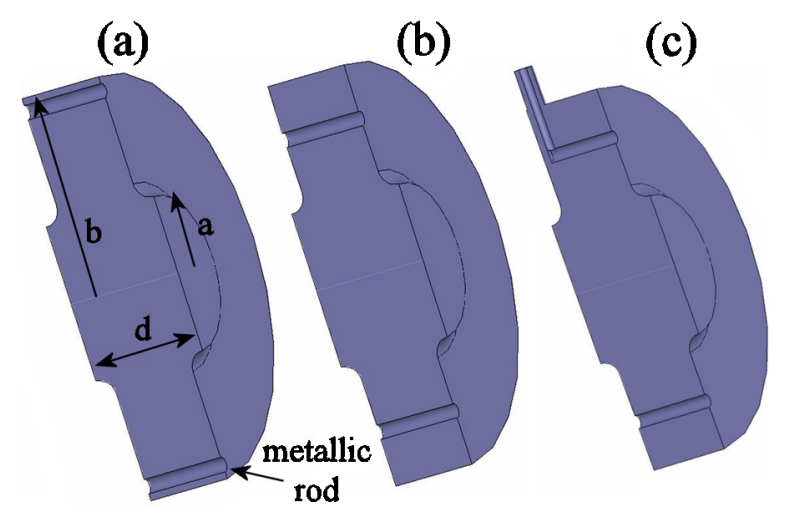

FIG. 11. Single cell geometry: (a) old; (b) rod modified; (c) rod modified and damping antenna. structure axis was $41 \mathrm{~mm}$ with a diameter of $4 \mathrm{~mm}$. For mechanical reasons the antenna loops have been located in opposite sides from one cell to the other. The dispersion curves are reported in Fig. 12. They have been calculated using the eigenmode module of HFSS and, in order to match the ports connected to the antenna, we have used the boundary condition "lumped RLC circuit" that well approximates the loads connection [8].

The quality factors of the vertical deflecting modes have been calculated simulating the structure shown in Fig. 13(a) with all ports matched. From simulations the quality factors were so low that they cannot be evaluated. The input/output couplers of the new structure have been redesigned using the technique discussed in [14] and are given in Fig. 13(b). We decided to maintain the same input/output coupler geometries of the previous realized deflectors [6] that did not give any problems at high input power. The other dimensions and in particular the iris diameters and the number of cells have been maintained equal to the previous realized deflectors [6].

Other possible solutions to shift the frequency of the vertical modes could be adopted. One possibility could be the use of large holes in the irises [15], another possibility could be the use of elliptical/asymmetric shapes of the cells. The first solution did not give large detuning for reasonable values of the hole dimensions while the second one was much more complicated because it was not possible to machine the cell by lathe. In our case, moreover, the rods have the function of antenna loops for mode damping.

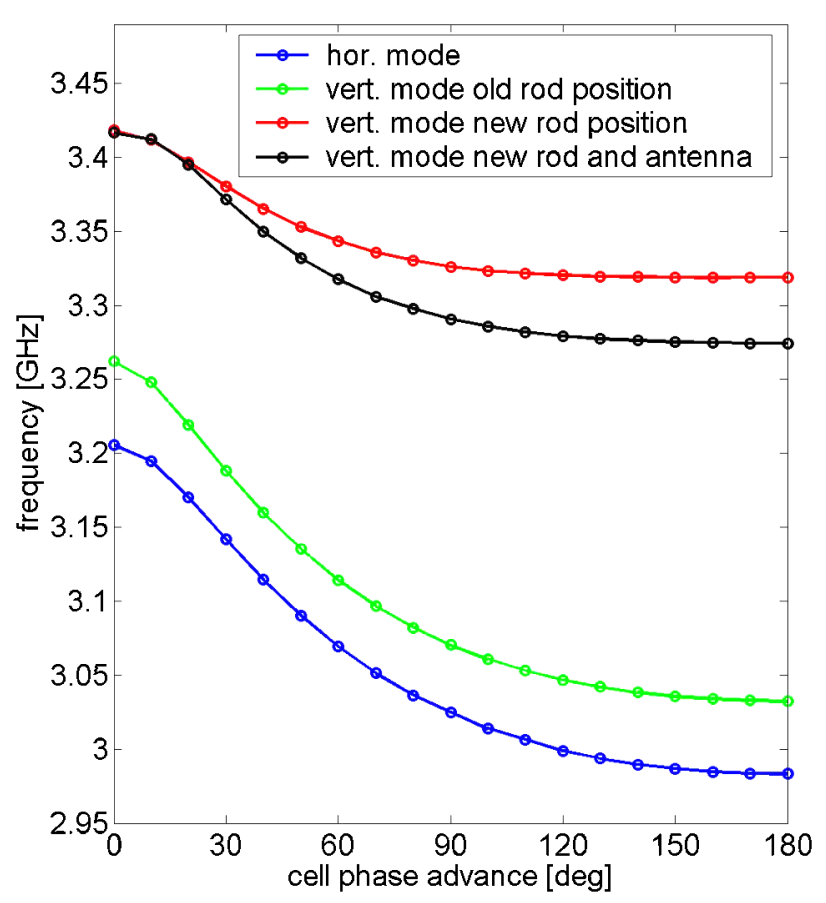

FIG. 12. Dispersion curves of the deflecting modes. 

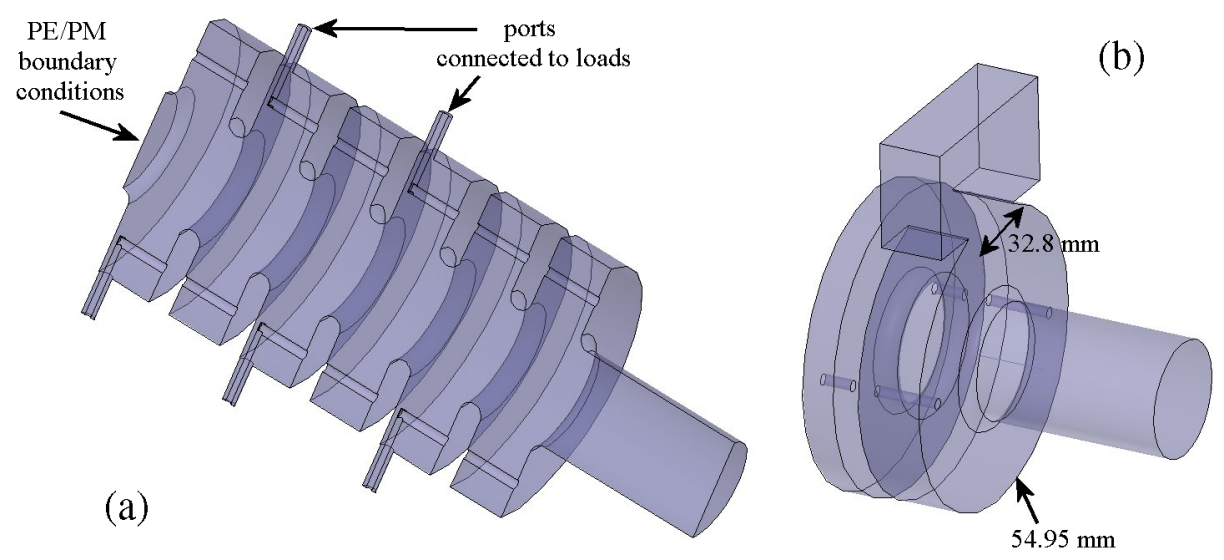

FIG. 13. HFSS geometries: (a) simulated structure to find the quality factor of the vertical modes; (b) coupler detail with dimensions.

Concerning the damping of the vertical polarity other possibilities were to adopt waveguides or particular shapes of the cells with absorbers [16]. These solutions were, in our opinion, much more complicated and expensive than the adopted one.

The last point that we have evaluated was the possible cross coupling between the main deflecting mode and the absorbing antennas. These could result, in principle, in a damage of the loads connected to the antennas and in a reduction of the horizontal deflecting kick for a given input power. While the second effect was less critical because in CTF3 we have enough power from the klystron, the first one was of more concern. The sources of this cross coupling could be: (a) the tilt of the cells along the structure; (b) the fact that the structure itself is not perfectly symmetric in both the horizontal and in the vertical planes: in the vertical plane, the asymmetries are represented by the antennas located in opposite sides from one cell to the other, while, in the horizontal plane, the asymmetries were due to the asymmetric couplers.

The first effect has been evaluated (at least to the first order) considering that the mode of the horizontal deflect- ing polarity has sine-type dependence with respect to the azimuthal angle. If the tilt of one cell is $\Delta \theta$, the power coupled from the antenna is a fraction $\sin (\Delta \theta)^{2}$ of the input power. Assuming a tilt $\Delta \theta \leq 0.5 \mathrm{deg}$ this fraction is less than $-40 \mathrm{~dB}$. With the $2 \mathrm{MW}$ nominal input power and the duty cycle of $1.5 \times 10^{-6}$ (see Table II), the average power into the loads would be $<3 \mathrm{~mW}$ while the peak voltage in the $1.5 \mu$ s pulse would be $<150 \mathrm{~V}$, both well below the loads and feedthrough limits.

The second effect has been evaluated simulating the structure with few cells. The cross coupling with the antenna nearest to the coupler has been $<-40 \mathrm{~dB}$ giving a tolerable power into the loads.

\section{DEFLECTOR REALIZATION AND RF TESTS}

Two plots of the final mechanical drawings are shown in Fig. 14. To reduce the cost and delivery time we built the new RFDs in aluminum. The cells have been machined, put into a stack, clamped, and welded together [17]. Figures 15 and 16 show the assembled cells and the final RFD under test. The tolerances of fabrication were $\pm 0.01 \mathrm{~mm}$ on the
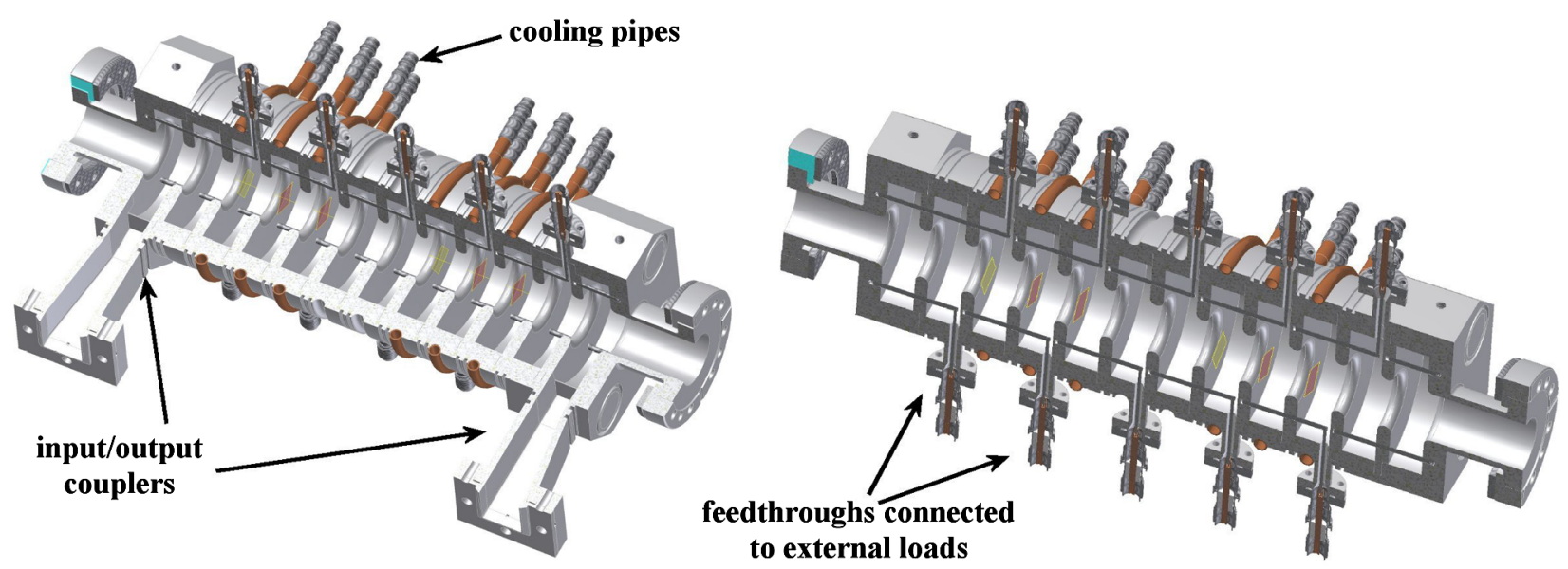

FIG. 14. Final mechanical drawings of the new RFDs. 


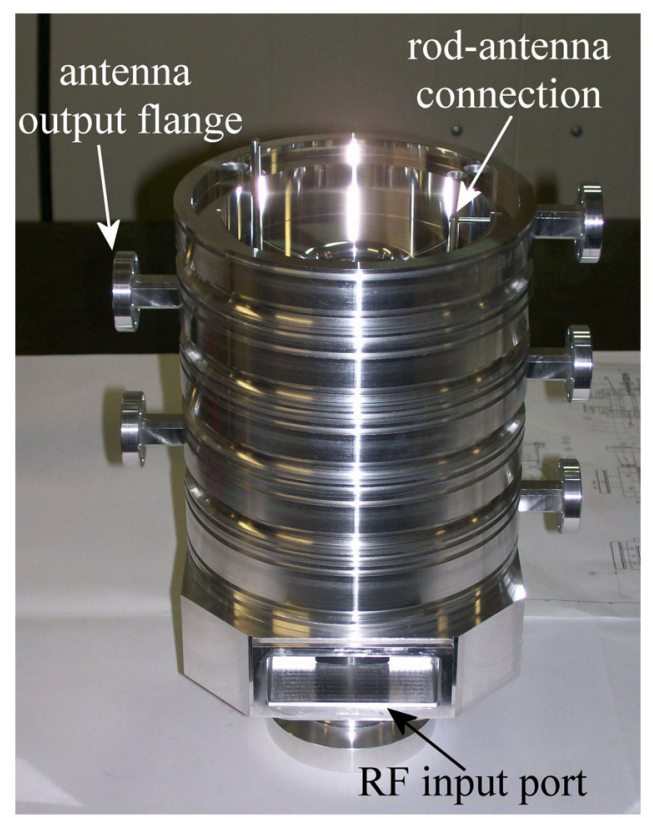

FIG. 15. Assembled RFD cells.

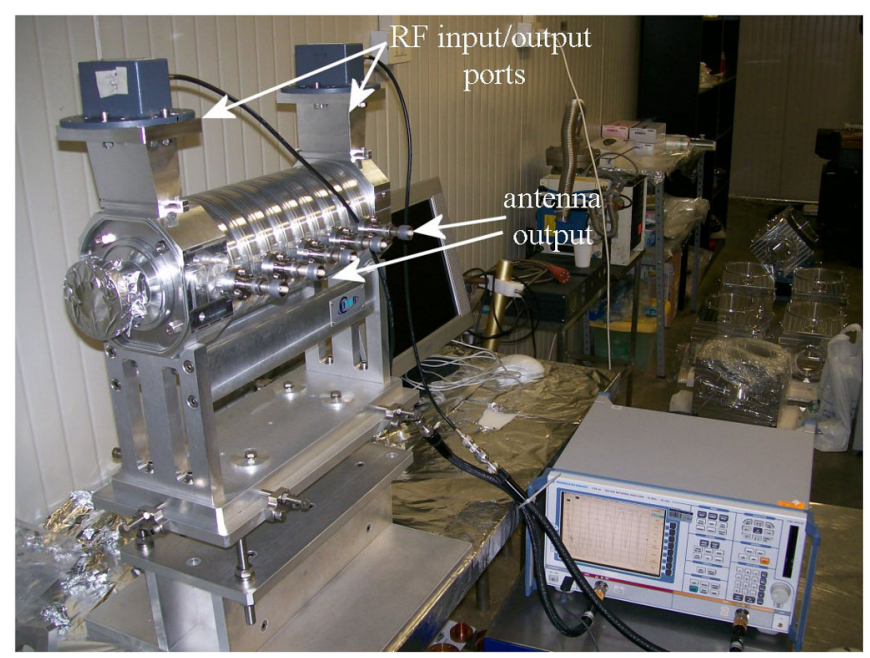

FIG. 16. RFD under test.

cell outer radius and $\pm 0.02 \mathrm{~mm}$ for the other dimensions. It is easy to verify that, under these conditions and in the worst case, one has a reduction of deflection $<1 \%$. This is the reason why we have decided to not tune the structures.

The rf measurements have been done in all phases of the construction to control the machining and the assembly process. The final measured reflection coefficient at the input port is reported in Fig. 17 and, at the working frequency, it was about $-25 \mathrm{~dB}$ as expected by simulations. The transmission coefficient between two antennas (the first and the last) is reported in Fig. 18 in two cases: all antennas connected to the loads and only two antennas (those used for measurements) connected to the loads.
According to the HFSS simulations, in the first case all modes have quality factors practically not measurable while in the second case some modes appear. However, also in this case, the damping is still very strong. ${ }^{6}$ Similar results can be obtained considering another pair of antennas.

Finally, the transmission coefficient between the rf input port and the antennas has been measured. As an example, the transmission coefficient between the input coupler and the antennas on the first and fifth TW cell is given in Fig. 19. As observed in the previous paragraph, a cross coupling has been measured since the structure is not perfectly symmetric in both the horizontal and in the vertical planes. From the measurements it is easy to note that the coupling is stronger in the first TW cell because of the proximity of the asymmetric coupler. Nevertheless, the couplings were below $-40 \mathrm{~dB}$ and guaranteed that the rf power does not couple significantly with the antenna loads causing reduction of the deflector efficiency and possible damages of the loads themselves.

Even if, from the multipacting (MP) point of view, the aluminum is worse with respect to the copper because of its higher secondary electron yield (SEY), the choice of the aluminum has been still maintained. The risks have been evaluated even if, at the beginning, no MP simulations were available. In particular, we were encouraged from the following reasons. First of all, no evidence of MP (even small) has been observed in the previously installed copper deflectors at any input power. Second, the dynamics of the secondary emitted electrons in deflecting structures is completely different with respect to the dynamics in accelerating structures and, in the region where we have maximum electric field, it is strongly perturbed by the high magnetic field. Third, in the case of strong MP, a backup solution could be to provide a Ti coating of the internal surfaces of the structure to reduce the SEY.

All these intuitive arguments have been confirmed by the very good performances of the deflectors and by MP simulations that we have done a posteriori by the code ANALYST [18]. First results are reported and discussed in [13].

\section{OPERATIONAL PERFORMANCES}

The detailed CR operation with the new RFD is beyond the scope of this paper and is widely described in $[19,20]$. Here it is important to remark that the cavities have been installed in the CR (August 2008), rf power conditioning needed less than one hour, and each cavity has been fed

\footnotetext{
${ }^{6}$ The modes are multicell modes and the loads connected to the two antennas are sufficient to give a strong damping. The $Q_{\text {ext }}$ of the modes that appear at $\approx 3.4$ and $\approx 3.6 \mathrm{GHz}$, when only two antennas are connected to the loads, are of the order of 200-300 and are related to higher order modes (like TE-like modes) with a very low $R / Q$.
} 


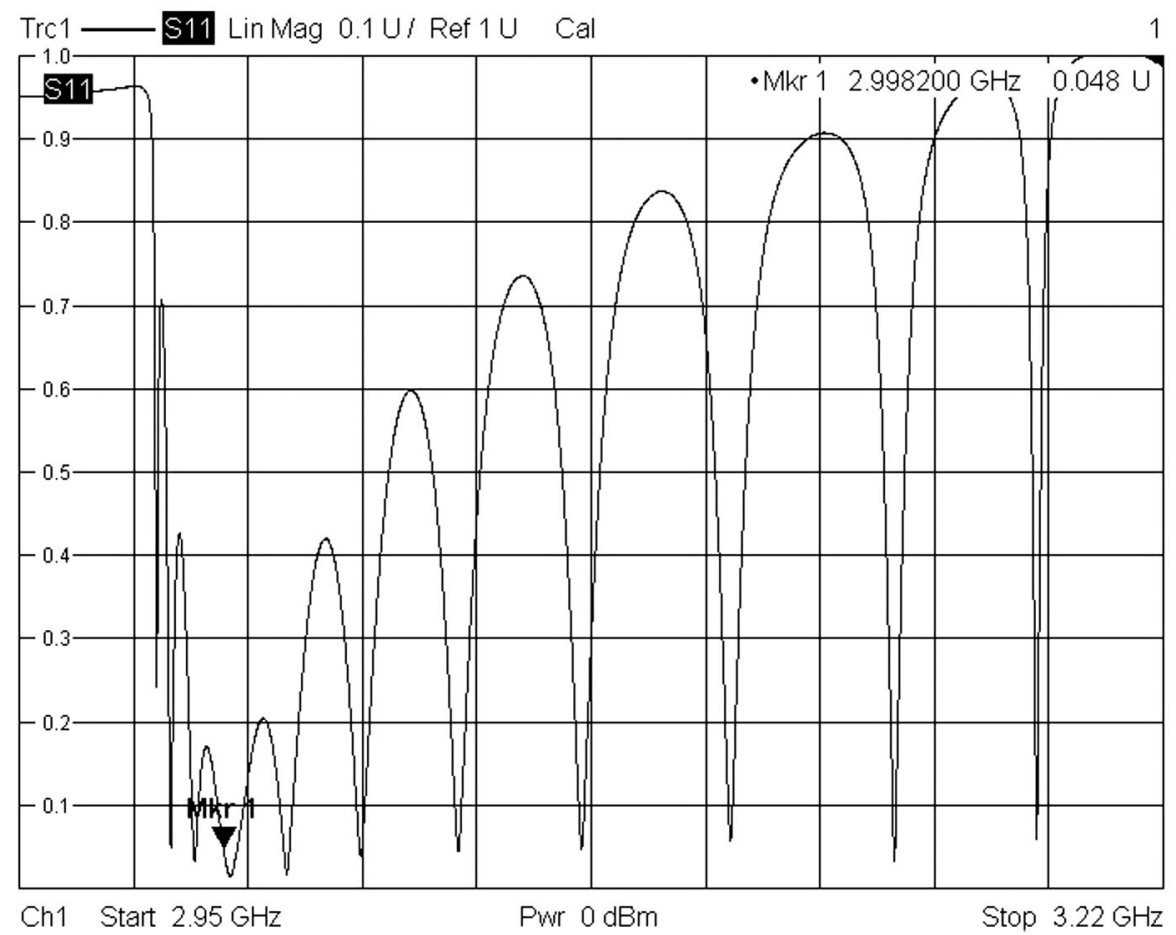

FIG. 17. Measured reflection coefficient at the input port.

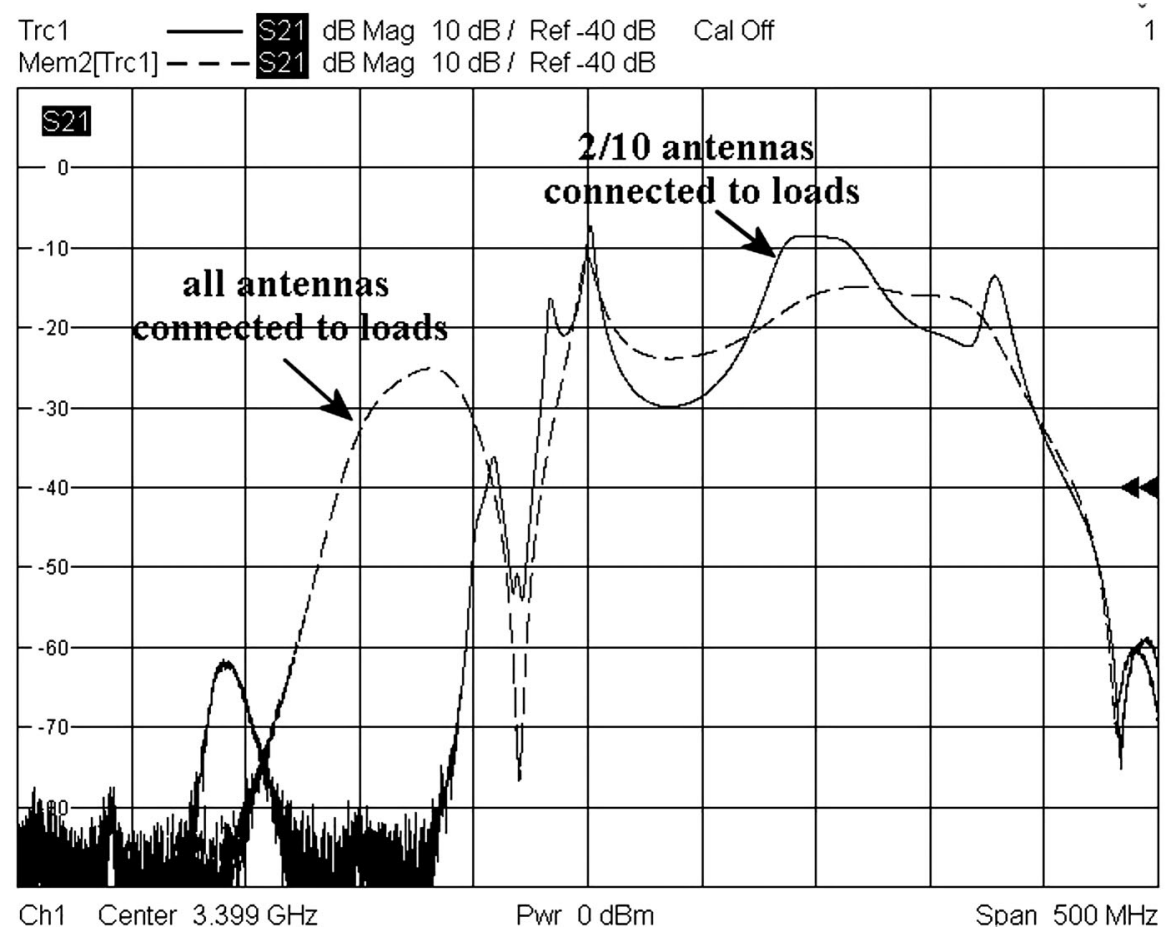

FIG. 18. Transmission coefficient between two antennas.

with more than $10 \mathrm{MW}$. Beam recombination in the CR has been achieved very soon without evidence of vertical instabilities $[19,20]$. As an example the scope traces from a ring BPM are reported in Fig. 20. This plot has to be compared with the previous one of Fig. 3. The fast growing oscillation disappeared and the beam could circulate without instabilities. The residual oscillations in the vertical and horizontal planes are given by small optics mismatches (like closed orbit,...) and have been (and are continuously) optimized during the further CTF3 runs. 
(a)

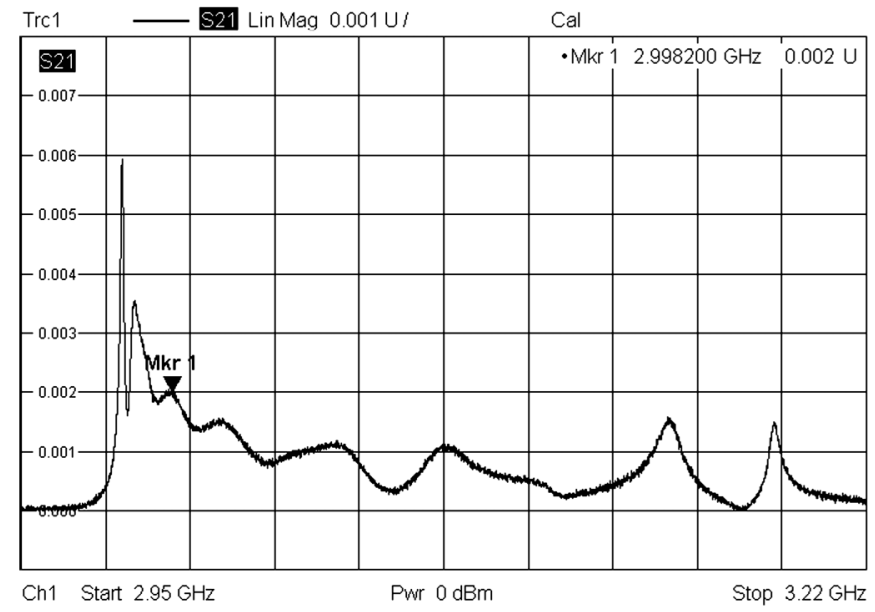

(b)

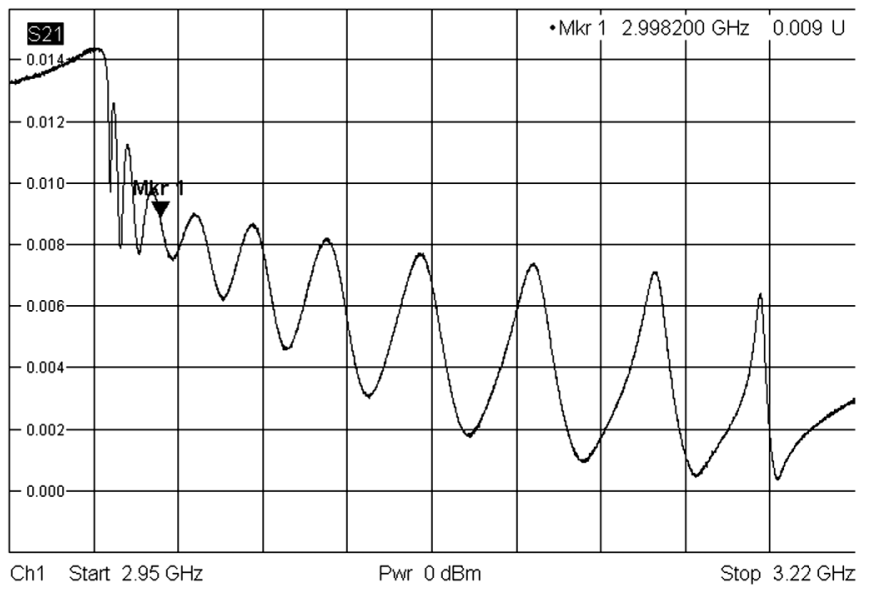

FIG. 19. Transmission coefficient between the input coupler and the antennas on the fifth (a) and first (b) TW cell.

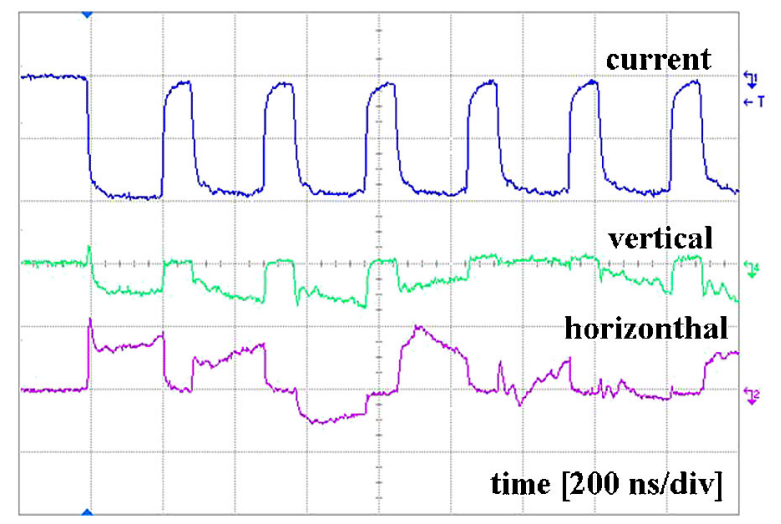

FIG. 20. Scope traces from a ring BPM after the installation of the new RFDs. The plot shows, from top to bottom, the circulating beam current in the case of 400 bunches and the vertical and horizontal signals. The vertical scale for the current signal is $\approx$ $2 \mathrm{~A} /$ division, the vertical scale for the vertical and horizontal signal is $\approx 2 \mathrm{~mm} /$ division.

\section{CONCLUSIONS}

Vertical trapped modes in the RFDs of the CTF3 CR have been identified to be the cause of the vertical beam instability found in the November 2007 run. A dedicated tracking code has been written to study in detail the beam dynamics and the effects of the ring and RFD parameters in the mitigation of the instability. The mechanism of the instability was similar to the beam breakup in a linear accelerator or in an energy recovery linac and the results of the code allowed identifying the main key parameters driving such instability and allowed finding the main knobs to mitigate it. In particular, the instability could be reduced by a more accurate control of the orbit inside the RFDs, by adopting a half integer vertical tune of the CR or by reducing the vertical $\beta$ function at the RFDs. Concerning the RFDs, the instability could be completely canceled with a strong reduction of the $Q$ factors of the vertical modes and with a strong detuning of such modes with respect to the horizontal working polarity.

For these reasons new RFDs have been designed and constructed. ${ }^{7}$ In these new devices the frequency separation between vertical and horizontal deflecting modes has been increased by modifying the splitting-mode rod positions in each cell and the rods themselves have been modified to become special antennas that absorb the power released by the beam to the vertical modes. The deflectors have been made in aluminum to reduce the costs and delivery time. Accurate low power rf tests have been done and have confirmed the expected results in terms of mode damping. The new structures have been successfully installed in the ring demonstrating the suppression of the instability itself. No multipacting effect has been found even if the structures have been realized in aluminum. A first preliminary analysis done a posteriori confirmed that in RFD traveling wave structures the multipacting is much less critical with respect to accelerating cavities.

\section{ACKNOWLEDGMENTS}

We would like to thank G. Fontana for the technical support in RFDs design and construction and G. McMonagle for the technical support in high power rf tests. We would like also to thank the CTF3 team at CERN for the beam measurements and instability characterization and $\mathrm{G}$. Geshonke and I. Syratchev for helpful discussions in the design of the new deflectors. Finally, we would like to acknowledge the personnel of the COMEB Company for its support and helpfulness in the deflectors' realization.

\footnotetext{
${ }^{7}$ On the other hand, the other proposed solutions to reduce the instability (such as the reduction of the vertical $\beta$ function at the RFDs, half integer vertical tune, ...) were less effective and also not practical from the operational point of view, while the new deflectors allow much more flexibility in the ring optics choice also.
} 
[1] CTF3 Design Report, CERN PS 2002-008 (RF), Geneve, 2002.

[2] J. Delahaye et al., in Proceedings of the 6th European Particle Accelerator Conference, Stockholm, 1998 (IOP, London, 1998).

[3] H. Braun et al., CERN Report No. 99-06, Geneve, 1999.

[4] J.P. Delahaye, CLIC07 Workshop, http://indico.cern.ch/ conferenceOtherViews.py?view=standard confId; $=17870$.

[5] R. Corsini et al., Phys. Rev. ST Accel. Beams 7, 040101 (2004).

[6] D. Alesini et al., in Proceedings of the 2002 European Particle Accelerator Conference, Paris (EPS-IGA/CERN, Geneva, 2002), p. 2115.

[7] R. Corsini et al., in Proceedings of the 11th European Particle Accelerator Conference, Genoa, 2008 (EPS-AG, Genoa, Italy, 2008), p. 574.

[8] http://www.ansoft.com

[9] See, for example, D. Alesini and F. Marcellini, Phys. Rev. ST Accel. Beams 12, 031301 (2009).

[10] J. J. Bisognano and G. A. Krafft, in Proceedings of the 1986 Linear Accelerator Conference, Stanford, CA (SLAC Report No. SLAC-303, 1986), pp. 452-454.

[11] Georg H. Hoffstaetter and Ivan V. Bazarov, Phys. Rev. ST Accel. Beams 7, 054401 (2004).
[12] D. Alesini et al., in Proceedings of the 11th European Particle Accelerator Conference, Genoa, 2008 (Ref. [7]), p. 574.

[13] D. Alesini et al., in Proceedings of the 23rd Particle Accelerator Conference, Vancouver, Canada, 2009 (IEEE, Piscataway, NJ, 2009).

[14] D. Alesini et al., Nucl. Instrum. Methods Phys. Res., Sect. A 580, 1176 (2007).

[15] O. H. Altenmueller et al., Report No. SLAC-R-017, 1963.

[16] G. Burt et al., in Proceedings of X-Band Structures and Beam Dynamics Workshop (XB08), 44th ICFA Beam Dynamics Workshop, Cockcroft Institute, UK, 2008, http://www.slac.stanford.edu/spires/find/hep?key= 8206180.

[17] The construction has been done at COMEB Company (http://www.comeb.it).

[18] I. Gonin et al., in Proceedings of the 2007 Particle Accelerator Conference, Albuquerque, New Mexico (IEEE, Albuquerque, New Mexico, 2007), pp. 2248-2250.

[19] S. Bettoni et al., Proceedings of the 23rd Particle Accelerator Conference, Vancouver, Canada, 2009 (Ref. [13]).

[20] F. Tecker, Progress in CTF3, CLIC Workshop 2009, CERN, Geneva, Switzerland, http://indico.cern.ch/ conferenceDisplay.py? confId $=45580$. 